\title{
HERBERT SUKOPP
}

\section{Selbstverpflichtungen der Wirtschaft als Instrument der Umweltpolitik}

Umweltpolitik ist immer zugleich Umweltschutz-Politik wie Umweltnutz-Politik. Umweltpolitik soll die Nutzung der in der Umwelt verfügbaren Ressourcen in Einklang bringen mit deren Erhaltung für zukünftige Generationen. Die übergeordneten Gemeinwohlziele sind letztlich nicht umstritten: die menschliche Gesundheit zu schützen und das Leben auf der Erde zu bewahren.

Vorsorgender Gesundheitsschutz ist ebenso wie das meist ressourcenökologisch und ressourcenökonomisch verstandene Leitbild der Nachhaltigkeit eine handlungsleitende Regel der dauerhaft umweltgerechten Entwicklung.

Selbstverpflichtungen der Wirtschaft gegenüber dem Staat sind ein Mittel zur Umsetzung des in der deutschen Umweltpolitik anerkannten und praktizierten Kooperationsprinzips.

Der folgende Text gibt die Stellungnahme des Rates von Sachverständigen für Umweltfragen wieder.

Bereits in den "Leitlinien Umweltvorsorge" der Bundesregierung aus dem Jahre $1986^{2}$ wurde zur Verwirklichung der Vorsorgepolitik die Unterstützung aller gesellschaftlicher Gruppen und der einzelnen Bürger gefordert. Sie ist eine Bedingung für die langfristige Umstrukturierung hin zu umweltschonenden Produktionsprozessen und Produkten. Kooperationen gewinnen an Bedeutung, weil es zur Verwirklichung eines ökologischen Strukturwandels noch stärker als bisher auf ein abgestimmtes Vorgehen aller verantwortlichen Akteure und auf die Ausnutzung von deren spezifischen Stärken ankommt und hoheitlichen Vorgaben in Form von ordnungsrechtlichen oder fiskalischen Instrumenten engere Grenzen gesetzt werden.

Die Selbstverpflichtungen der Industrie sind auf relativ große Beachtung gestoßen, weil man in ihnen einen richtungsweisenden Trend für die Umweltpolitik der kommenden Jahre zu erkennen glaubte. Befürworter sprachen von einem Durchbruch auf dem Weg zu einer Umweltpolitik der Arbeitsteilung zwischen Staat und Wirtschaft; Skeptiker befürchten einen beginnenden Erosionsprozeß staatlicher Verantwortung bei der

Vgl. SRU 1996, 1998. Zur weiteren Entwicklung vgl. ELNI 1998, Engel 1998, Kloepfer 1998.

2 BT-Drs. 10/6028. 
Festlegung umweltpolitischer Ziele. Entscheidend für eine Bewertung derartiger Lösungen sind aus Sicht des Umweltrates in erster Linie die ökologische Effektivität und die Akzeptanz in der Bevölkerung. Daneben sind auch ökonomische, rechtliche und administrative Bezüge von Bedeutung. In der Vergangenheit waren ausschlaggebend für den Erfolg derartiger Vereinbarungen in erster Linie der Druck der Öffentlichkeit und drohende ordnungsrechtliche Maßnahmen.

\section{Bestandsaufnahme}

\subsection{Ausmaß des Einsatzes von Selbstverpflichtungen}

Seit etwa Mitte der siebziger Jahre werden in der deutschen Umweltpolitik Selbstverpflichtungen als ergänzendes Instrument eingesetzt. Seit Anfang der achtziger Jahre hat die deutsche Wirtschaft etwa 80 Selbstverpflichtungen (einschließlich einiger ,formeller" Vereinbarungen) abgegeben; seit Mitte 1998 gab es keine neuen Selbstverpflichtungen. In der Koalitionsvereinbarung für die 14. Legislaturperiode des Deutschen Bundestages heißt es dazu:

„Das Instrument der freiwilligen Selbstverpflichtung stärkt das Umweltbewußtsein der Akteure. Sie können insbesondere in klar abgegrenzten Bereichen sinnvoll sein und zu effektivem Umwelthandeln beitragen, wenn die zu erreichenden Ziele und Zwischenziele eindeutig festgelegt und überprüfbar (Monitoring) sind und sie im Falle der Nichteinhaltung mit Sanktionen verbunden werden. Selbstverpflichtungen in dieser Form können auf geeignetem Gebiet Ordnungsrecht entbehrlich machen, z.B. als Vereinbarung mit Unternehmen. Die Umwelthaftungspflicht wird entsprechend ausgebaut."

Die Zahl der Selbstverpflichtungen ist (im europäischem Vergleich, wo nur die Niederlande auf eine höhere Zahl kommen) relativ hoch, wobei es in den Jahren bis 1998 einen erheblichen Anstieg gegeben hat. Sie betreffen insbesondere das Einstellen der Herstellung bestimmter Produkte oder der Verwendung bestimmter Stoffe in Produkten (Asbest, FCKW in einer Anzahl von Geräten und in Hartschäumen, wassergefährliche Stoffe in Thermo- und Durchschreibepapieren, Inhaltsstoffe von Wasch- und Reinigungsmitteln), die Erfassung und Bewertung von chemischen Zwischenprodukten, die Kennzeichnung und Anwender- und Behördeninformationen für Wasch- und Reinigungsmittel sowie bestimmte Textilbehandlungsmittel, den Entsorgungsbereich (Batterien, Verpackungen, FCKW in bestimmten Geräten, Papier, Altautos, elektronische Abfälle und Bauschutt), die Einleitung von Stoffen in Gewässer (Ammonium, EDTA, Zellstoffherstellung), $\mathrm{CO}_{2}$-Emissionen (Reduzierung der $\mathrm{CO}_{2}$-Emissionen und Erhöhung der Energieeffizienz in einer Reihe von Sektoren, Kraftstoffverbrauch von Kraftfahrzeugen), Methanemissionen (Begrenzung der Methanemissionen in der Aluminiumindustrie) sowie den Rohstoffverbrauch bei der Herstellung und beim Konsum (Papierherstellung, Waschmittel).

Seit Anfang der neunziger Jahre haben Selbstverpflichtungen im Bereich der Abfallwirtschaft zur Umsetzung der Produktverantwortung der Hersteller und des Handels eine besondere Rolle erlangt. Die bedeutendste Selbstverpflichtung ist allerdings - im Hin- 
blick auf den bisher weitgehend unüblichen Regelungsgegenstand (Emissionsreduzierung), die große Reichweite und ihre Verknüpfung mit einer zentralen Zielsetzung der modernen Umweltpolitik - die Erklärung der deutschen Industrie zur Reduzierung der $\mathrm{CO}_{2}$-Emissionen und Steigerung der Energieeffizienz aus dem Jahre 1995, die im Jahre 1996 modifiziert und ausgeweitet wurde. Sie umfaßt nunmehr 19 verschiedene Branchen der deutschen Wirtschaft, auf die etwa siebzig Prozent des Energieverbrauchs der Industrie und beinahe die gesamte öffentliche Energieversorgung entfällt; im Hinblick auf die Teilnahme der Verbände der deutschen Gas- und Elektrizitätsversorgungsindustrie und der kommunalen Unternehmen betrifft sie mittelbar auch die meisten Haushalte und kleingewerblichen Verbraucher. Daneben gibt es eine ergänzende Selbstverpflichtung der deutschen Automobilindustrie für die Verminderung des spezifischen Kraftstoffverbrauchs von Personenkraftfahrzeugen (1990, modifiziert im Jahre 1994).

Überwiegend sind Selbstverpflichtungen auf nationaler Ebene abgegeben worden, jedoch gibt es auch eine Reihe von Verpflichtungen auf Landesebene.

\subsection{Struktur von Selbstverpflichtungen}

Die Struktur der Selbstverpflichtungen ist recht unterschiedlich. Zum Teil gibt es relativ einfache Formen, die beispielsweise in einem kurzen Text ein Versprechen der entsprechenden Branche enthalten, die Verwendung eines bestimmten Einsatzstoffes ganz oder teilweise, sofort oder allmählich zu beenden. Zum Teil handelt es sich dabei um eine vorgezogene Einstellung der Verwendung eines Stoffes, der zu einem späteren Zeitpunkt durch Verordnung verboten wird; oder um Stoffe, die überhaupt nicht durch Ordnungsrecht erfaßt sind. Komplexer sind Selbstverpflichtungen, insbesondere, wenn Substitutstoffe noch nicht für alle Verwendungszwecke vorhanden sind. In diesen Fällen wird die Selbstverpflichtung ,weicher" formuliert, mit Angaben zu Forschungs- und Entwicklungsarbeiten sowie Berichtspflichten gegenüber dem zuständigen Umweltminister oder dem Umweltbundesamt.

Die Bezeichnung „freiwillige Selbstverpflichtung“, die öffentlichkeitswirksam gewählt wird, ist ungenau, weil die Erwartung einer staatlichen Regelung häufig eine Voraussetzung für den Abschluß von Selbstverpflichtungen ist. ${ }^{3}$

\subsection{Rechtsformen von Selbstverpflichtungen}

Im Bereich der Umweltpolitik in Deutschland ist die Selbstverpflichtung die übliche Rechtsform konzertierter Selbstregulierung, nicht die „formelle“ Vereinbarung. Die Selbstverpflichtung wird in der Form einseitiger Erklärungen oder Empfehlungen nationaler Branchenverbände, gegebenenfalls auch eines nationalen Industrieverbandes abgegeben. Bisweilen verpflichten sich auch einzelne Unternehmen (oder beteiligen sich an Selbstverpflichtungen). Das zuständige Ministerium ist nicht formell beteiligt, jedoch erfolgen in aller Regel intensive Diskussionen oder gar Verhandlungen mit den zuständigen Ministerien (Umweltministerium, in gewissem Umfang auch Wirtschafts-

\footnotetext{
3 Vgl. Kap. 2.2.
} 
ministerium, in der Vergangenheit auch Gesundheitsministerium) und bisweilen mit dem Umweltbundesamt. Die Verpflichtungserklärungen werden an das zuständige Ministerium gerichtet und von diesem regelmäßig informell ,anerkannt", zum Beispiel durch eine Presseerklärung. Die zuständigen Ministerien üben vielfach ein erhebliches $\mathrm{Maß}$ an Kontrolle über die Erreichung der festgelegten Ziele aus. Deshalb enthalten beinahe alle Selbstverpflichtungen Berichtspflichten der Wirtschaft gegenüber dem Staat, sehen in Deutschland aber keine Sanktionen vor (aber z.B. in den Niederlanden und Belgien).

\subsection{Gründe für den Einsatz von Selbstverpflichtungen}

Die wachsende Bedeutung von Selbstverpflichtungen in der Umweltpolitik hat zwei miteinander zusammenhängende politische Gründe. Zum einen ist es erklärte Politik der Bundesregierung, Selbstverpflichtungen möglichst den Vorrang vor ordnungsrechtlichen Lösungen zu geben. Diese Position entspricht weitgehend den Forderungen der Wirtschaft, während sie von den Umweltverbänden entweder strikt abgelehnt oder doch mit erheblicher Skepsis bewertet wird. Insoweit sind sich die Umweltverbände mit jenen Wissenschaftlern und Politikern einig, die ordnungspolitische Bedenken gegen Selbstverpflichtungen erheben.

Um das Aufkommen von Selbstverpflichtungen zu erklären, kann auch auf Ergebnisse sozialwissenschaftlicher Forschungen zur Stellung des Staates in der Gesellschaft zurückgegriffen werden, wonach die Beziehungen zwischen Staat und Gesellschaft schon lange nicht mehr ausschließlich durch hierarchische staatliche Kontrolle gekennzeichnet sind. Vielmehr ist durch politikwissenschaftliche Forschungen zunehmend deutlich geworden, daß die Koordination zwischen staatlichen und gesellschaftlichen Akteuren in einer Vielzahl von Formen geleistet wird, vor allem in Verhandlungssystemen zwischen staatlichen und privaten Akteuren. Die allgemein zunehmende Bedeutung solcher Verhandlungssysteme für die Koordination staatlichen Handelns hängt mit grundsätzlichen Entwicklungen im Verhältnis von Staat und Gesellschaft zusammen. Einerseits übernimmt die Politik in modernen Demokratien immer neue und immer komplexere Aufgaben. Andererseits nimmt die Fähigkeit des Staates, gesellschaftliche Prozesse zentral und hierarchisch zu steuern, durch strukturelle Entwicklungen in der modernen Gesellschaft ab. Der Staat sieht sich im Ergebnis also Aufgaben gegenüber, die mit traditionellen Koordinationsmechanismen nicht mehr zu bewältigen sind. Die in den letzten Jahrzehnten zunehmend institutionalisierten Verhandlungssysteme zwischen staatlichen und gesellschaftlichen Akteuren reagieren präzise auf diese Probleme. ${ }^{4}$

In der Politikwissenschaft hat sich für diese Systeme der Begriff des korporatistischen Arrangements bzw. des Politiknetzwerkes durchgesetzt. Während korporatistische Systeme die beiden zentralen Konfliktparteien und den Staat umfassen, bestehen Netzwerke aus einer großen Zahl von Akteuren, die alle ein Interesse an einem Regelungsgegenstand haben. Netzwerkartige Strukturen ermöglichen es ganz allgemein, das Problemlösungspotential aller Akteure zu nutzen und im Rahmen relativ stabiler und institutionali-

Vgl. Schuppert 1995; Mayntz 1993; Scharpf 1993; Böhret 1992. 
sierter Kooperationssysteme zu gemeinsamen Lösungen der komplexen und längerfristigen Probleme der Gesellschaft zu gelangen. Dies schließt auch die Formulierung und Durchführung der Umweltpolitik mit ein. Trotz allem bleibt der Staat in solchen Verhandlungsnetzwerken ein privilegierter Akteur, der das öffentliche Interesse vertritt und als Moderator fungiert. Er kann dabei stets auf hoheitlichen Zwang zurückgreifen, wenn das Politiknetzwerk unzureichende Ergebnisse hervorbringt. Staatliche Akteure können damit nach wie vor die Resultate solcher Verhandlungen maßgeblich beeinflussen. Die Regelungen, die in diesen Verhandlungssystemen getroffen werden, können verschiedene Formen annehmen. Selbstverpflichtungen als Ergebnis von intermediären Verhandlungen sind weder ein neues Phänomen noch sind sie auf umweltpolitische Problemstellungen beschränkt. Dennoch hat diese Form der Koordination in Verhandlungssystemen in den letzten Jahren gerade in der Umweltpolitik große Bedeutung erlangt.

Neben den Problemen der Effektivität und ökonomischen Effizienz des Instruments, ist vor allem die Frage nach der Legitimität solcher Verhandlungen zu stellen. Dabei ist zunächst von großer Bedeutung, daß die Verhandlungen zwischen staatlichen und wirtschaftlichen Akteuren um den Abschluß von Selbstverpflichtungen verfahrensmäßig nicht abgesichert sind. Damit fehlt ihnen die Legitimität von Regelungen, die in geordneten Gesetzgebungsverfahren beschlossen werden. Außerdem tendieren Selbstverpflichtungen, ähnlich wie klassische neokorporatistische Arrangements, zur Erzeugung von Kompromissen zu Lasten nichtbeteiligter Dritter. Es ist daher die Frage zu stellen, ob solche Arrangements zusätzlicher Partizipation etwa durch Umweltverbände oder ähnliche interessierte Akteure bedürfen.

\section{Vor- und Nachteile von Selbstverpflichtungen}

\subsection{Bewertungsgrundlagen}

Die Frage, wie Selbstverpflichtungen gegenüber administrativer Regulierung einerseits, ökonomischen Instrumenten andererseits, zu bewerten sind, wird heute zunehmend kontrovers diskutiert. $\mathrm{Zu}$ beachten ist, daß in der historischen Entwicklung dieses Instrument ausschließlich als Alternative zum Ordnungsrecht angesehen wurde und die Vor- und Nachteile auch heute noch überwiegend unter dieser Perspektive diskutiert werden. Indessen tritt in jüngerer Zeit die Selbstverpflichtung auch in Konkurrenz zu ökonomischen Instrumenten, wie dies insbesondere in der Klimapolitik deutlich geworden ist. Bei der Bewertung von Selbstverpflichtungen ist von ausschlaggebender Bedeutung, woran man ihre Akzeptabilität mißt. ${ }^{5}$ In Betracht kommt der Vergleich mit alternativen Instrumenten (Ordnungsrecht, ökonomische Instrumente), dem technischen Vermeidungspotential, der Entwicklung der Umweltbelastung aufgrund eines Trendszenarios (,business as usual“-Szenario), und der Umweltbelastung im Basisjahr (Vorher-Nachher-Vergleich). In der neueren Diskussion zur Bewertungsproblematik sind vor allem zwei methodische Defizite erkennbar.

\footnotetext{
5 Vgl. Europäische Umweltagentur 1997, S. 12, 52 ff.; Börkey/Glachant 1997, S. 35 ff.
} 
Einmal neigt man dazu, von einem bestimmten Realtypus der Selbstverpflichtung (z.B. Rücknahme- und Verwertungsverpflichtung oder Reduzierung von $\mathrm{CO}_{2^{-}}$ Emissionen) auszugehen und die für diesen Typ entwickelten Befunde zu verallgemeinern. Damit wird man der Typenvielfalt von Selbstverpflichtungen nicht gerecht. Abgesehen von grundsätzlichen ordnungspolitischen Bedenken ist weder eine pauschale Verurteilung noch eine generelle Bejahung von Selbstverpflichtungen sachlich angebracht.

Zum anderen ist eine Neigung unverkennbar, festgestellte oder vermutete Mängel von praktizierten oder projektierten Selbstverpflichtungen in der realen Welt mit den Vorteilen idealer ordnungsrechtlicher Regulierung oder idealer ökonomischer Instrumente zu vergleichen. ${ }^{6}$

Auf methodische Schwierigkeiten oder jedenfalls Datenprobleme stoßen aber auch die meisten anderen denkbaren Vergleichsmaßstäbe, insbesondere der Vergleich mit dem technischen Vermeidungspotential, mit dem Trendszenario und selbst mit dem Basisjahr. ${ }^{7}$ Insgesamt ist vor dem Hintergrund vieler Untersuchungen festzustellen, daß Aussagen über den Erfolg oder Mißerfolg von Selbstverpflichtungen erhebliche methodische Unschärfen aufweisen.

\subsection{Umweltwirksamkeit}

\subsubsection{Untersuchungen zu einzelnen Selbstverpflichtungen}

Die Umweltwirksamkeit der in der Vergangenheit abgegebenen Selbstverpflichtungen wird vielfach, wenngleich mit unterschiedlichen Akzentuierungen, positiv eingeschätzt. ${ }^{8}$ Allerdings fehlt es weitgehend an - methodisch abgesicherten - empirischen Untersuchungen. Als Gründe für das Scheitern von Selbstverpflichtungen werden die mangelnde Akzeptanz neuer Produkte beim Verwender, technische Schwierigkeiten bei der Produktsubstitution und mangelnde Homogenität der Anbietergruppen genannt.

Eine positive Einschätzung gilt insbesondere für produktbezogene Verpflichtungen (Beendigung des Einsatzes von Asbest, APEO, FCKW sowie Kennzeichnung und Verbraucherinformationen bei Wasch- und Reinigungsmitteln). Es gibt allerdings wichtige Ausnahmen. Zum Beispiel hat die Selbstverpflichtung der Lackindustrie zur Einstellung der Verwendung von Schwermetallen und Lösemitteln in Lacken die gesetzten Ziele nur zur Hälfte erfüllt, da zwar Schwermetalle weitgehend eliminiert wurden, die industriellen und gewerblichen Verwender - im Gegensatz zu den privaten Verbrauchern aber lösemittelfreie Lacke bisher vielfach nicht akzeptiert haben. Im übrigen dürfte der Erfolg mancher Selbstverpflichtungen, die einen vorzeitigen Ausstieg aus der Verwendung bestimmter Stoffe vorsehen, zum Teil auch darauf zurückzuführen sein, daß die beteiligte Branche überlange Anpassungsfristen für sich erreichen konnte. Eine empirische Studie, die die Umweltwirksamkeit und die hierfür maßgeblichen Faktoren für einige Selbstverpflichtungen aus den achtziger Jahren (Asbest, FCKW in Aerosolver-

6 Vgl. ZEW 1998, S. 90 f., 181, 210, 219, 243.

Vgl. Europäische Umweltagentur 1997, S. 12 ff., 52 ff., 83 ff.

8 Vgl. Troge 1997; Clausen/Brokmann/Schellenberger 1995, S. 16; Lautenbach/Steger/Weihrauch 1992. 
packungen, Schwermetalle und Lösemittel in Lacken und APEO in Wasch- und Reinigungsmitteln) aufgrund eines Vorher-Nachher-Vergleichs untersucht hat, kommt zu dem Ergebnis, daß mit der teilweisen Ausnahme der Selbstverpflichtung der Lackindustrie (beschränkt auf Lösemittel) alle anderen Selbstverpflichtungen sehr erfolgreich gewesen sind, wobei allerdings die identifizierten Gründe recht unterschiedlich waren. ${ }^{9}$

\subsubsection{Allgemeine Bewertungskriterien}

Für die Umweltwirksamkeit von Selbstverpflichtungen sind Prozeß und Ergebnis sowohl der Zielfindung als auch der Zielerreichung von Bedeutung. Im Schrifttum werden Selbstverpflichtungen idealtypisch danach unterschieden, ob das zu erreichende Ziel bereits feststeht, oder ob über das Ziel noch verhandelt werden muß, wie das insbesondere bei produktbezogenen Selbstverpflichtungen der Fall sei. ${ }^{10}$ Im ersten Fall hätten Staat und Wirtschaftsverband das gleiche Interesse an einer Minimierung der Kosten insgesamt für die Zielerreichung, jedoch seien zwischen den Mitgliedsfirmen Verhandlungen über die Verteilung der Lasten erforderlich. Stehe dagegen das Ziel nicht fest, müßten zwischen Staat und Verband Verhandlungen über die Strenge des Ziels stattfinden; diese Verhandlungen seien konfliktreicher, ihr Erfolg setze eine glaubwürdige Drohung des Staates mit eigener Zielfestlegung voraus.

Dieses idealtypische Modell läßt jedoch außer acht, daß in jedem Fall ein gewisser Spielraum hinsichtlich der Zielfestlegung besteht, da die einer Selbstverpflichtung zugrunde gelegten Umweltziele kaum jemals rechtlich bindend fixiert sind. Insofern ist eine glaubwürdige Erwartung einer staatlichen Regelung durch Ordnungsrecht oder ökonomische Instrumente eine Grundvoraussetzung für das Zustandekommen von Selbstverpflichtungen mit anspruchsvollen Umweltzielen - jedenfalls wenn sie per saldo mit erheblichen Kostenbelastungen und sonstigen Nachteilen verbunden sind Auch wenn der Staat, soweit er von vornherein den Weg der Selbstverpflichtung anvisiert, seine Verhandlungsposition schwächt, ${ }^{11}$ hat er das Ordnungsrecht und ggf. auch ökonomische Instrumente stets in der Hinterhand. Die Glaubwürdigkeit der Drohung mit staatlicher Regelung dürfte - unabhängig von einer Vorfestlegung auf Selbstverpflichtungen - in erster Linie vom umweltpolitischen Klima und den dadurch begründeten Chancen auf eventuelle Durchsetzung der Zielvorstellungen im politischen Prozeß abhängen.

Umgekehrt trifft auch die von den meisten Akteuren vertretene Auffassung, die Zielfestlegung sei Sache des Staates, ${ }^{12}$ kaum zu. Nur die rechtliche Letztverantwortlichkeit für die Zielfestlegung aufgrund von Verhandlungen muß beim Staat liegen; sie manifestiert sich spätestens bei der Entgegennahme der Selbstverpflichtung und ist besonders deutlich, wenn die Bundesregierung eine Selbstverpflichtung durch eine Verordnung flankiert, wie das bei den neueren abfallwirtschaftlichen Selbstverpflichtungen der Fall ist. Insofern ist die ordnungspolitisch motivierte Kritik am Instrument der Selbstver-

\footnotetext{
9 Vgl. Lautenbach/Steger/Weihrauch 1992.

10 Vgl. Europäische Umweltagentur 1997, S. 14, 86; Glachant 1994a; Glachant 1994b.

11 Vgl. ZEW 1998, S. 153.

12 Vgl. COWI 1997, S. 34 ff.
} 
pflichtung, der Staat entziehe sich seiner Politikverantwortung, ${ }^{13}$ nicht stichhaltig. Was bleibt, ist die von Behördenvertretern bemerkte Einschränkung, Selbstverpflichtungen könnten wegen ihres Tauschcharakters zu einer Verwässerung oder Verschiebung beim Erreichen staatlicher Umweltziele führen, die aus der Sicht einer angemessenen Umweltpolitik in bestimmter Weise gesetzt oder durchgesetzt werden sollten. Kritiker ${ }^{14}$ bringen als scheinbar überzeugungskräftiges Beispiel, daß sich die deutsche Wirtschaft nur zu einer Reduzierung der spezifischen $\mathrm{CO}_{2}$-Emissionen um $20 \%$ verpflichtet habe, während das Ziel der Bundesregierung bei $25 \%$ liege. Die allgemeine Gültigkeit dieses Arguments ist schwer zu beurteilen, da in der bisherigen Umweltpolitik quantifizierte Ziele kaum je festgesetzt wurden. Es ist auch nicht feststellbar, welches Ergebnis beim Versuch einer ordnungsrechtlichen Regulierung oder des Einsatzes ökonomischer Instrumente erzielt worden wäre. ${ }^{15}$ Eine rechtlich bindende Zielfestlegung existiert nur bei Stoffverboten, die im Wege der Selbstverpflichtung vorzeitig erfüllt werden. Hier besitzt die Selbstverpflichtung anscheinend einen eindeutigen Vorteil gegenüber der administrativen Regelung, jedoch bleibt zweifelhaft, ob es nicht möglich gewesen wäre, knappere Übergangsfristen zu setzen. Auf jeden Fall erscheint es nicht sinnvoll, Selbstverpflichtungen wegen mangelnder Zielkonformität zu kritisieren, weil sie nur Ziele anstreben, die denen eines vorliegenden Verordnungsentwurfs entsprechen, der seinerseits wegen zu weitgehender Ziele politisch stark umstritten ist. ${ }^{16}$ Auch die Befürchtung, Selbstverpflichtungen orientierten sich regelmäßig am schwächsten Glied, ${ }^{17}$ erscheint jedenfalls nicht verallgemeinerungsfähig. Sie ist beispielsweise nicht stichhaltig, wenn, wie bei der Klimaschutzerklärung der deutschen Industrie, das Gesamtziel die Summe der von den beteiligten Branchen und Unternehmen übernommenen Reduzierungsleistungen ist.

Welche Vorteile haben Selbstverpflichtungen aus der Sicht der Umweltadministration? Staatliche Umweltziele können schneller politisch durchgesetzt werden, und durch das Moment der Freiwilligkeit können auf lange Sicht bessere Ergebnisse erzielt werden als durch eine ordnungsrechtliche Regelung. Dagegen spricht allerdings die lange Verhandlungsdauer bei komplexen Selbstverpflichtungen. Der Erwartung besserer Ergebnisse steht das Argument gegenüber, die Wirtschaft verspreche im Wege der Selbstverpflichtung regelmäßig nicht mehr, als dies der langfristigen wirtschaftlichen und technologischen Entwicklung - unabhängig von staatlicher Intervention - entspreche.

Die gegenwärtige Methodenentwicklung reicht noch keineswegs aus, um gesicherte Aussagen über die Umweltwirksamkeit von Selbstverpflichtungen zu machen. Die meisten Selbstverpflichtungen sind noch zu jungen Datums, als daß ihre Umweltwirksamkeit abschließend bewertet werden könnte. Soviel zur Zielfindung.

Hinsichtlich der Zielerreichung bestehen ebenfalls Zurechnungsprobleme, da die erreichte Umweltverbesserung auch durch nicht von der Selbstverpflichtung induzierte wirtschaftliche Entwicklungen, zum Beispiel Produktionseinbußen, oder als Folge zu-

\footnotetext{
13 Vgl. ZEW 1998, S. 96, 166, 189, 216.

14 Siehe Holzhey/Tegner 1996 und DIW 1995.

$15 \mathrm{Vgl}$. Europäische Umweltagentur 1997, S. 41 f.; Börkey/Glachant 1997, S. 37.

16 So aber ZEW 1998, S. 224 f., 249 zur Altautoerklärung.

17 Vgl. Monopolkommission 1996, Tz. 96.
} 
sätzlich eingesetzter Anreize verursacht worden sein kann. Unabhängig hiervon sind die vielfach anzutreffende mangelnde Reichweite und die Rechtsform bloßer Selbstverpflichtungen ohne rechtliche Bindungswirkung von Nachteil. Selbstverpflichtungen umfassen nicht immer alle Wettbewerber, so daß das Problem besteht, daß Außenseiter die Vereinbarung unterlaufen können. Dies gilt insbesondere für ausländische Anbieter. Außerdem kann es für die Erreichung der Ziele der Selbstverpflichtung von Bedeutung sein, auch nachgelagerte Wirtschaftsstufen einzubeziehen, um den Erfolg der von den Initiatoren der Selbstverpflichtung angestrebten Produktsubstitution sicherzustellen.

Ein weiterer, freilich unterschiedlich bewerteter Mangel liegt in der „Weichheit“" von Selbstverpflichtungen. Mangels rechtlicher Verbindlichkeit und Verfügbarkeit von Sanktionen für Staat und beteiligten Industrieverband kann Trittbrettfahrerverhalten seitens der Mitgliedsfirmen rechtlich nicht verhindert werden. Soweit in der Vergangenheit Selbstverpflichtungen gescheitert oder nur unzulänglich erfüllt worden sind, lag dies vielfach an anderen Umständen (z.B. fehlende Einbeziehung aller Verwendergruppen, mangelnde Akzeptanz neuer Produkte durch die Abnehmer oder völlige Veränderung der Kalkulationsgrundlagen für alle beteiligten Unternehmen). Lediglich die Selbstverpflichtung über Getränkeverpackungen (1977/1982) ist ein eindeutiger Beleg für das Scheitern einer Selbstverpflichtung aufgrund Trittbrettfahrerverhaltens. In diesem Bereich liegen freilich sehr ungünstige Voraussetzungen für den Erfolg einer Selbstverpflichtung vor. ${ }^{18}$

Insgesamt läßt sich daher eine allgemeine Aussage dergestalt, daß hinsichtlich ihrer Umweltwirksamkeit Selbstverpflichtungen ordnungsrechtlichen Lösungen stets eindeutig unterlegen sind, kaum treffen. Entsprechendes gilt wegen der Schwierigkeit der Festsetzung ,richtiger" Abgabensätze auch im Verhältnis zu Abgabenlösungen. Dagegen ist die Zielerreichung bei Zertifikaten - abgesehen von illegalem, durch Straf- und Ordnungsrecht zu ahndendem Verhalten - durch die Struktur des Instruments sichergestellt.

Als ein entscheidender Testfall wird bisweilen die Klimaschutzerklärung der deutschen Wirtschaft gesehen, die einerseits die Chance auf den Einstieg in eine andere, d.h. auf Selbstverantwortung aufbauende Vorsorgepolitik biete, andererseits auch das Risiko in sich berge, daß bei ihrem Scheitern das Instrument der Selbstverpflichtung politisch diskreditiert sei. ${ }^{19}$ Dies stellt insofern eine unzulässige Verallgemeinerung dar, als ein Mißerfolg dieser Selbstverpflichtung allenfalls die Untauglichkeit emissionsbezogener Selbstverpflichtungen belegen könnte, der Einsatz im Produktbereich und in der Abfallwirtschaft jedoch damit nicht präjudiziert ist.

\subsection{Allokative Effizienz}

Ein häufiges, aber umstrittenes Argument zugunsten von Selbstverpflichtungen ist die Annahme, daß diese zu einer Verbesserung der allokativen Effizienz führen. Empirische

\footnotetext{
$18 \mathrm{Vgl}$. IFO 1997, S. 163.

19 Siehe dazu Schafhausen 1997, S. 196 f.
} 
Daten, die eine Effizienzanalyse gestatten, stehen regelmäßig nicht zur Verfügung. ${ }^{20}$ Allerdings gibt es für die Effizienz alternativer Instrumente, insbesondere von Umweltabgaben, ebenfalls keine empirischen Belege. ${ }^{21}$ Effizienzvorteile liegen theoretisch vor allem in der Möglichkeit einer Differenzierung des Umfangs und der Zeit der Durchführung von Beschränkungen nach der unterschiedlichen Grenzkostenstruktur von Branchen und Unternehmen und nach Investitionszyklen sowie in der Möglichkeit von Optimierungslösungen für das ganze Unternehmen. ${ }^{22}$ Ordnungsrechtliche Regelungen sind notwendigerweise abstrakt-generell und können nicht ohne weiteres die Besonderheiten einzelner Branchen und individueller Unternehmen berücksichtigen. Selbstverpflichtungen erreichen in dieser Sicht ein Ausmaß an Differenzierung, das die Interessen und die Struktur der betroffenen Wirtschaft in angemessenem Umfang berücksichtigt. Es erscheint evident, daß Regelungen von der Art der Klimaschutzerklärung der deutschen Industrie mit ihrem hohen Grad an Differenzierung nach Branchen durch eine Verordnung nicht hätten erreicht werden können. Auch die Struktur der abfallwirtschaftlichen Selbstverpflichtungen dürfte anders sein, als eine alternativ mögliche ordnungsrechtliche Regelung dies vorgesehen hätte.

Es gibt jedoch gewichtige Gegenargumente: Zunächst geht jedenfalls bei emissionsbezogenen sowie bei stoffbezogenen Selbstverpflichtungen, soweit diese nur eine Reduzierung des Einsatzes von Inhaltsstoffen in Produkten - und nicht deren völligen Ausschluß - anstreben, ein Gutteil der Effizienzvorteile verloren, wenn eine Verteilung der Reduzierungs- und sonstigen Anpassungslasten auf die einzelnen Branchen und Mitgliedsfirmen entsprechend deren unterschiedlichen Grenzkosten nicht stattfindet. $^{23}$ Explizite Verteilungsregeln zwischen den Unternehmen innerhalb einer Branche gibt es in der Verbandsrealität offenbar ebenfalls nicht. Als Gründe werden hierfür insbesondere Gesichtspunkte des Wettbewerbs angeführt. Ausgleichend wirken insoweit allerdings die großzügig bemessenen Anpassungsfristen, die es jedem beteiligten Unternehmen erlauben, den kostengünstigsten Zeitpunkt für eigene Maßnahmen zu wählen. Überdies ist zu berücksichtigen, daß sich, wie bei der Klimaschutzerklärung, die Verbandsziele oft aus der Summe der von den einzelnen Unternehmen angebotenen, wirtschaftlich für vertretbar gehaltenen Reduzierungs- oder Einsparungspotentialen zusammensetzen; in diesem Fall ist eine entsprechende Implementation mit daraus folgenden Effizienzvorteilen zu erwarten. Gleichwohl bleibt die Logik der verbandsinternen Verteilung der Lasten von Selbstverpflichtungen ein gut gehütetes Geheimnis, und die Wirtschaft wehrt sich, eine Verteilung der übernommenen Reduzierungslasten durch Einrichtung eines internen Lizenzmarktes, der das Effizienzkriterium am besten erfüllen würde, ${ }^{24}$ durchzuführen. Effizienzverluste treten auch durch die mit Selbstverpflichtungen vielfach verbundenen Wettbewerbsbeschränkungen sowie durch Trittbrettfahrer ein. ${ }^{25}$

${ }^{20}$ Vgl. Europäische Umweltagentur 1997, S. 42, 72, 91.

21 Vgl. Europäische Umweltagentur 1996, S. 29.

22 So Wicke/Knebel 1997, S. 16 f.; vgl. auch COWI 1997, S. 34 ff.; IEA 1997, S. 40 f.

23 Vgl. Börkey/Glachant 1997, S. 40; ZEW 1998, S. 217; DIW 1995, S. 279.

${ }^{24}$ Siehe dazu IFO 1997, S. 164 ff.

25 Vgl. IEA 1997, S. 4I; ZEW 1998, S. 100, 174. 


\subsection{Innovationswirkungen}

Besonders umstritten scheinen die Auswirkungen von Selbstverpflichtungen auf die technologische Innovation zu sein. Eine allgemeine Bewertung ist schwierig, weil Innovation von einer Reihe von Faktoren wie Industriestruktur, Entwicklungsphase des betreffenden Unternehmens und bestehenden Techniklinien abhängig ist, die von Selbstverpflichtungen nicht ohne weiteres zu beeinflussen sind. ${ }^{26}$ Erfahrungen aus der Vergangenheit zeigen, daß einzelne Selbstverpflichtungen technologische Innovationen in Gang gesetzt haben. ${ }^{27}$ Andererseits ergeben empirische Untersuchungen, daß die Wirtschaft selbst den Einfluß von Selbstverpflichtungen auf die technologische Innovation nicht besonders hoch einschätzt. ${ }^{28}$ Man wird davon ausgehen können, daß Selbstverpflichtungen, die die Einstellung der Verwendung bestimmter Stoffe oder Verfahrensänderungen vorsehen, für die betroffene Wirtschaft jedenfalls dann zu riskant sind, wenn die technologische Innovation im betreffenden Bereich noch in Gang oder noch nicht absehbar ist. Sie können jedoch einen bereits in Gang befindlichen oder absehbaren Innovationsprozeß beschleunigen.

\subsection{Systemkonformität}

Gibt der Staat mit der Anerkennung von Selbstverpflichtungen seine Verantwortung für das Gemeinwohl auf, einen umweltbezogenen Ordnungsrahmen für das System der Gesellschaftssteuerung durch den Markt zu geben? Besteht die Gefahr von Wettbewerbsbeschränkungen sowie der Unternehmenskonzentration? Ferner befürchtet man die Preisgabe des politischen Handlungsspielraums des Staates sowie eine Interventionsspirale, wenn die Selbstverpflichtung scheitern sollte. ${ }^{29}$ Obwohl diese Einwände nicht in allen Punkten überzeugen, hat die ordnungspolitische Kritik so viel Gewicht, daß ein vorsichtiger Umgang mit Selbstverpflichtungen anzuraten ist. Selbstverpflichtungen verschieben die Aufgabenverteilung im Verhältnis zwischen Staat und Markt. Der Staat gibt mit der Akzeptierung von Selbstverpflichtungen seine Gemeinwohlverantwortung allerdings nicht auf, sondern erfüllt sie nur in anderer Form. Deshalb ist es auch nicht angebracht, von einer Privatisierung der Zielfestlegung zu sprechen. Eine Beteiligung der Wirtschaft am Zielfindungsprozeß gibt es unabhängig vom jeweils eingesetzten Instrument. Die größere Intensität der Teilhabe an diesem Prozeß bei Selbstverpflichtungen wird durch Zugang des Staates zur knappen Ressource Information ausgeglichen.

Die Frage, ob Partizipation der Öffentlichkeit, insbesondere von wirtschaftlich betroffenen Dritten, Verbraucher- und Umweltorganisationen, am Verhandlungsprozeß angestrebt werden sollte, ist bisher stark umstritten, und es gibt gewichtige Argumente für beide Positionen. Die Europäische Kommission befürwortet entsprechend dem nie-

26 Vgl. Europäische Umweltagentur 1997, S. 43 ff;; Börkey/Glachant 1997, S. 41.

27 So in den Fällen von Asbest, FCKW, APEO, EDTA

28 Vgl. COWI 1997, S. 34 ff.

29 Vgl. ZEW 1998, S. 96, 166, 189, 216; ferner z.B. Burchardi/Sacksofsky 1994, S. 31 f. 
derländischen Modell einen Ausbau der Partizipation, ohne sich freilich zu deren Formen (bloße Stellungnahme zu einem fortgeschrittenen Entwurf, Teilnahme an den Verhandlungen, Erfordernis eines Konsenses) präzise zu äußern. Gegen eine Partizipation hinsichtlich eines fortgeschrittenen Entwurfs einer Selbstverpflichtung lassen sich begründete Einwände kaum geltend machen. Dies bedeutet freilich nicht, daß eine Verrechtlichung der Partizipation erfolgen müßte. Darüber hinaus sollte ein angemessenes $\mathrm{Maß}$ an nachträglicher Partizipation hinsichtlich des Erfolgs von Selbstverpflichtungen gewährleistet sein.

Es ist allgemein anerkannt, daß Transparenz ein unerläßliches Kriterium jedenfalls für die Akzeptanz von Selbstverpflichtungen ist. ${ }^{30}$ Zum Teil wird sogar eine verbindliche Transparenzerfordernis für Selbstverpflichtungen aus dem Demokratiegebot abgeleitet. ${ }^{31}$ Die Texte der wichtigeren neueren Selbstverpflichtungen sind grundsätzlich öffentlich zugänglich, und es gibt zum Teil auch umfangreiche Presseberichte über sie. Eine vollständige Textveröffentlichung erfolgt selten. In vielen Fällen ist es sogar schwierig festzustellen, welcher Branchenverband überhaupt an einer Selbstverpflichtung beteiligt ist. In bezug auf die Ergebnisse, das heißt die Zielerreichung, ist die Transparenz noch weitaus geringer entwickelt. Erst in jüngeren Selbstverpflichtungen ist vorgesehen, daß die Ergebnisse des Monitoring auch der Öffentlichkeit zugänglich gemacht werden. In diesem Zusammenhang ist darauf hinzuweisen, daß Zugang zu den Ergebnissen eines Monitoring wohl nicht aufgrund des Umweltinformationsgesetzes erreicht werden kann, da dieses Gesetz vom Zugangsrecht solche Informationen ausschließt, die ein Unternehmen der Behörde freiwillig gegeben hat. ${ }^{32}$ Der Umweltrat schlägt die Errichtung einer Clearingstelle vor, die sowohl die Texte als auch die Monitoring-Ergebnisse der Öffentlichkeit zugänglich macht. Darüber hinaus sollte die Bundesregierung gehalten sein, auf der Grundlage der Ergebnisse des Monitoring sowie der sonstigen Berichterstattung den Erfolg von Selbstverpflichtungen und gegebenenfalls Abhilfemaßnahmen periodisch zu prüfen und einer öffentlichen Anhörung zu unterwerfen, jedenfalls in Politikfeldern, in denen nach den gesetzlichen Regelungen für Verordnungen und Verwaltungsvorschriften eine Anhörung der beteiligten Kreise vorgeschrieben ist. Zwar ist davon auszugehen, daß Erfolge und Mißerfolge von Selbstverpflichtungen auch im Parlament und in der Presse diskutiert werden und die politische Letztverantwortung der Exekutive dadurch aktualisiert werden kann. Jedoch dienen Transparenz und nachträgliche Partizipation der Kompensation für den Ausfall der vorherigen Öffentlichkeits- und Verbändebeteiligung. Sie haben eine wichtige Vorfeldwirkung in Richtung auf umweltpolitisch vertretbare Selbstverpflichtungen und erhöhen die politische Aufmerksamkeit als unerläßliche Voraussetzung für öffentliche Kontrolle, aber auch Akzeptanz von Selbstverpflichtungen.

Im Umweltgutachten 1998 des Umweltrates werden weiterhin wettbewerbspolitische und wettbewerbsrechtliche Probleme sowie verfassungs- und europarechtliche Fragestellungen besprochen. Wegen der erheblichen ordnungspolitischen Bedenken und Bewertungsprobleme erscheint dem Umweltrat ein vorsichtiger, eher restriktiver Einsatz

\footnotetext{
30 Troge 1997, S. $149 \mathrm{f}$.

31 So Brohm 1992, S. 1031.

$32 \S 7$ Abs. 4 UIG.
} 
des Instruments Selbstverpflichtung nach wie vor geboten; vor einer weiteren Klärung der Bewertungsprobleme und der Gewinnung ausreichender praktischer Erfahrungen dürfen Selbstverpflichtungen nicht zu einem zentralen Instrument der Umweltpolitik werden. Ihre verfassungsrechtliche Zulässigkeit und die verfassungsrechtlichen Grenzen sind keineswegs gesichert. Sie liegen vielmehr in einer rechtlichen "Grauzone“, was auch aus diesem Grund einen vorsichtigen Umgang mit diesem Instrument der Umweltpolitik nahelegt.

\section{Schlußfolgerungen}

Die Vorteile von Selbstverpflichtungen liegen zum einen darin, daß sie der staatlichen Umweltpolitik Zugriff auf Informationen geben, die nur bei der Wirtschaft vorhanden sind, und das Problemlösungspotential aller Akteure der Gesellschaft nutzen, um zu gemeinsamen Lösungen komplexer und langfristiger Umweltprobleme zu gelangen. Zum anderen können sie dazu beitragen, die negativen Verteilungswirkungen von Umweltschutzmaßnahmen abzumildern, indem sie Lösungen ermöglichen, die auf die Kostensituation und den Investitionszyklus der einzelnen Unternehmen zugeschnitten sind. Effizienzvorteile sind nur bedingt zu erwarten.

Produktbezogene Selbstverpflichtungen kommen in Betracht, wo weniger umweltbelastende Substitute noch nicht verfügbar sind, aber die absehbaren Innovationstrends für die Zukunft erwarten lassen, daß solche Substitute entwickelt werden können.

In jedem Fall bedarf es vor dem Einsatz der Selbstverpflichtung als Instrument der Umweltpolitik einer sorgfältigen Evaluation, besonders im Hinblick auf Wirksamkeit und Wettbewerbswirkungen. Sie müssen soweit wie möglich quantifizierte Ziele anstreben, die durch Zugrundelegung eindeutiger und nachprüfbarer Basisgrößen (z.B. Basisjahr, Basisemissionen) überprüfbar sind; sie müssen umweltpolitisch anspruchsvoll sein und daher über die nach dem regelmäßigen Verlauf der Entwicklung erwartbaren Umweltentlastungen hinausgehen; ihr Erfolg muß durch Monitoring und Berichte transparent gemacht werden.

Das weitgehende Fehlen von Möglichkeiten der Partizipation in der gegenwärtigen Praxis der Selbstverpflichtung sollte überwunden werden, ohne freilich eine Verrechtlichung des Instruments anzustreben. Der Öffentlichkeit, insbesondere den Umweltverbänden, sollte die Möglichkeit eingeräumt werden, zu Selbstverpflichtungen in einem fortgeschrittenen Entwurfsstadium Stellung zu nehmen. Die Bundesregierung sollte ferner gehalten sein, periodisch den Erfolg und ggf. die notwendigen Maßnahmen bei absehbarem Mißerfolg einer Selbstverpflichtung zu prüfen und einer Öffentlichkeitsbeteiligung zu unterwerfen. Hierdurch kann das Partizipationsdefizit bei Verhandlungen über Selbstverpflichtungen kompensiert, die Letztverantwortung der Bundesregierung für den Erfolg ihrer Umweltpolitik verdeutlicht und Akzeptanz für das Instrument der Selbstverpflichtung gewonnen werden. 


\section{Literaturverzeichnis}

Böhret, C. (1992), Zur Handlungsfähigkeit des funktionalen Staates der spätpluralistischen Industriegesellschaft, in: Staat und Demokratie in Europa, hg. v. B. Kohler-Koch, Opladen, S. 116-129.

Börkey, P./Glachant, M. (1997), Les engagement volontaire de l'industrie dans le domaine de l'environnement: nature et diversité, Paris.

Brohm, W. (1992), Rechtsgrundsätze für normersetzende Absprachen, in: Die Öffentliche Verwaltung 46, S. 1025-1035.

Burchardi, W./Sacksofsky, E. (1994), Wettbewerbspolitische und kartellrechtliche Probleme der deutschen Entsorgungswirtschaft, in: Jahrbuch für Umwelt- und Technikrecht, S. 25-30.

Clausen, J./Brokmann, F./Schellenberger, O. (1995), Selbstyerpflichtungsabkommen im Umweltschutz, Berlin (Institut für ökologische Wirtschaftsforschung, Diskussionspapier 36/95).

COWI [Consulting Engineers and Planners AS] (1997), Study on Voluntary Agreements Concluded Between Industry and Public Authorities in the Field of the Environment. Final Report, Kopenhagen.

DIW [Deutsches Institut für Wirtschaftsforschung] (1995): „Selbstverpflichtung“ der Wirtschaft zur $\mathrm{CO}_{2}$-Reduktion: Kein Ersatz für aktive Klimapolitik, in: DIW-Wochenbericht 62, S. 277-283.

ELNI [Environmental Law Network International] (Hg., 1998), Environmental Agreements. Role and Effects of Environmental Agreements in Environmental Policies, Den Haag u.a.

Engel, C. (1998), Selbstregulierung im Bereich der Produktverantwortung. Instrumente und deren Ausgestaltung, Bonn.

EUA [Europäische Umweltagentur] (1996), Environmental Taxes - Implementation and Environmental Effectiveness, Kopenhagen (European Environmental Agency).

EUA [Europäische Umweltagentur] (1997), Environmental Agreements. Environmental Effectiveness, Kopenhagen (European Environmental Agency. - Environmental Issues Series Nr. 3).

Glachant, M. (1994a), Les Accordes Volontaires dans la Politique Environmentale: Une Mise en Perspective de leur Nature et de leur Efficacité, Paris (Centre d'Economie Industrielle), in: Economie et Prévisions, i. E.

Glachant, M. (1994b), Voluntary Agreements in Environmental Policy (Report prepared for the Environment Directorate of OECD [unveröffentl.]).

Holzhey, M./Tegner, H. (1996), Selbstverpflichtungen - Ein Ausweg aus der umweltpolitischen Sackgasse?, in: Wirtschaftsdienst 76, S. 425-430.

IEA [International Energy Agency] (1997), Voluntary Actions for Energy-Related $\mathrm{CO}_{2}$ Abatement, Organisation for Economic Cooperation and Development, Paris.

IFO [Institut für Wirtschaftsforschung] (1997), Förderung sinnvoller Getränkeverpackungen. Endbericht, Berlin (Umweltbundesamt, UBA-Texte 17/97).

Kloepfer, M. (Hg., 1998), Selbst-Beherrschung im technischen und ökologischen Bereich, Berlin.

Knebel, J. (1999), Selbstverpflichtungen und normersetzende Umweltverträge als Instrumente des Umweltschutzes, in: UBA (Umweltbundesamt), Reihe Berichte, Bd. 5/99.

Lautenbach, S./Steger, U./Weihrauch, P. (1992), Evaluierung freiwilliger Branchenvereinbarungen (Kooperationslösungen) im Umweltschutz, in: Bundesverband der Deutschen Industrie: Freiwillige Kooperationslösungen im Umweltschutz. Ergebnisse eines Gutachtens und Workshops, Bundesverband der Deutschen Industrie, BDI-Drucksache 249, Köln, S. 1-166.

Leitlinien Umweltvorsorge (1986), Leitlinien der Bundesregierung zur Umweltvorsorge durch Vermeidung und stufenweise Verminderung von Schadstoffen, BT-Drs. 10/6028.

Mayntz, R. (1993), Policy-Netzwerke und die Logik von Verhandlungssystemen, in: Policy-Analyse. Kritik und Neuorientierung, Politische Vierteljahresschrift, So.-H. 24, hg. v. A. Héritier, Opladen, S. 39-56. 
Monopolkommission (1996), Wettbewerbspolitik in Zeiten des Umbruchs: Hauptgutachten XI, BadenBaden.

Schafhausen, F. (1997), Die Erklärung der deutschen Wirtschaft zur Klimavorsorge: Instrumentenmix als Ansatz für eine nachhaltig wirksame Umweltvorsorgepolitik, in: Wicke 1997, S. 171-200.

Scharpf, F. (1993), Positive und negative Koordination in Handlungssystemen, in: Policy-Analyse. Kritik und Neuorientierung, Politische Vierteljahresschrift, So.-H. 24, hg. v. A. Héritier, Opladen, S. 57-83.

Schuppert, G. F. (1995), Rückzug des Staates? Zur Rolle des Staates zwischen Legitimationskrise und politischer Selbstbestimmung, in: Die Öffentliche Verwaltung 48, S. 761-770.

SRU [Der Rat von Sachverständigen für Umweltfragen bei dem Bundesminister für Umwelt, Naturschutz und Reaktorsicherheit] (1996): Umweltgutachten 1996, Stuttgart.

SRU [Der Rat von Sachverständigen für Umweltfragen bei dem Bundesminister für Umwelt, Naturschutz und Reaktorsicherheit] (1998): Umweltgutachten 1998, Stuttgart.

Troge, A. (1997), Erfolgs- und Problemfälle umweltbezogener Selbstverpflichtungen aus der Sicht des Umweltbundesamts, in: Wicke 1997, S. 133-151.

Wicke, L./Knebel, J. (1997), Umweltbezogene Selbstverpflichtungen der Wirtschaft: Chancen und Grenzen für Umwelt, (mittelständische) Wirtschaft und Umweitpolitik, in: Wicke 1997, S. 1-50.

Wicke, L. (Hg., 1997), Umweltbezogene Selbstverpflichtungen der Wirtschaft - umweltpolitischer Erfolgsgarant oder Irrweg?, Bonn.

ZEW [Zentrum für europäische Wirtschaftsforschung] (1998), Umweltstandards im internationalen Handel, Materialien zur Umweltforschung Nr. 32, Stuttgart. 


$$
\text { t }
$$

